\title{
Electron Tomography Contributing to Ultrastructural Research in Vascular Biology and Transfusion Medicine
} Josef Neumüller ${ }^{1,2}$, Thomas Wagner ${ }^{3 *}$

'Blood Donation Center of the Austrian Red Cross for Vienna, Lower Austria and Burgenland

${ }^{2}$ Center for Anatomy and Cell Biology, Department for Development Biology, Medical University of Vienna

${ }^{3}$ Department of Blood Group Serology and Transfusion Medicine, Medical University of Graz, Austria

Article Info

\section{Article Notes}

Received: January 25, 2019

Accepted: February 16, 2019

\section{*Correspondence:}

Prof. Dr. Thomas Wagner, Department of Blood Group Serology and Transfusion Medicine, Medical University of Graz, Austria; Email: thomas.wagner@medunigraz.at.

C 2019 Wagner T. This article is distributed under the terms of the Creative Commons Attribution 4.0 International License.

\section{Keywords}

Electron Tomography (ET)

Transmission Electron Microscopy (TEM)

Endothelial Progenitor Cells (EPC)

Platelet concentrates

Bacterial contamination

Transfusion medicine.

\section{Abstract}

In this review, the technique of electron tomography is shortly summarized. Alignment procedures on the electron microscope are described. New techniques such as the focused ion beam tomography, the block face scanning tomography and the array tomography are mentioned. Application of these methods for vascular research, concerning endothelium-specific WeibelPalade bodies in cell cultures from human umbilical vein endothelial cells and endothelial progenitor cells as well as about cellular communications between the endothelium and the subendothelium are referred. Platelets are important players in the interaction with the endothelium in thrombosis and hemostasis after transfusion, therefore electron tomographic investigations of platelet organelles as well as platelet microparticles are mentioned. Illustrations from our own investigations are added.

Abbreviations: ART: Algebraic Reconstruction Technique; ADAMTS-13: A Disintegrin And Metalloproteinase with A Thrombospondin Type 1 Motif, Member 13; CCD Camera: Charge Coupled Device Camera; DAB: 3,3'-Diaminobenzidine; DART: Discrete Algebraic Reconstruction Technique; DTS: Dense Tubular System; EM: Electron Microscope; EPC: Endothelial Progenitor Cells; ET: Electron Tomography; FVIII: Factor VIII Blood Clotting Protein; FIB-SEM: Focused Ion Beam Scanning Electron Microscope; FITC: Fluorescein-Isothiocyanate; HUVEC: Human Umbilical Vein Endothelial Cells; LSM: Laser Confocal Scanning Microscope; MIT: Mitochondria; MTC: Microtubular Coil; OCS: Surface-Connected Open Canalicular System; PLT: Platelets; PLTC: Platelet Concentrates; PMP: Platelet Microparticles; ROI: Region Of Interest; SBEM: Serial Block Face Scanning Electron Microscopy; SIRT: Simultaneous Itinerative Reconstruction Technique; STEM: Scanning Transmission Electron Microscopical Device; TEM: Transmission Electron Microscope; TGN: Trans Golgi Network; TRITC: TetramethylrhodamineIsothiocyanate; vWF: von Willebrand Factor; WPB: Weibel Palade Bodies; WPB: Method: Weighted Back Projection Method.

\section{Introduction}

A recently published review ${ }^{1}$ gave rise to an invitation to author a mini-review about electron tomography (ET) in vascular biology and transfusion medicine. ET was introduced in the last decade of the last century ${ }^{2}$. We established this method in our Department for Cell Biology and Ultrastructure Research (head: Margit Pavelka) at the Medical University of Vienna, Austria in 2004, encouraged by the work of three powerful research groups (Wofgang Baumeister, at the Department 
of Molecular Structural Biology at the Max Plank Institute for Biochemistry, Martinsried, Germany; Abraham J. Koster, Department of Molecular Cell Biology, Faculty of Biology Institute of Biomembranes, Utrecht - now head of the Section of Electron Microscopy, Department of Molecular Cell Biology, University of Leiden, The Netherlands and Kathryn E. Howell, University of Colorado School of Medicine, Department of Cell and Development Biology, USA). Our main emphasis was placed on the dynamic response of the Golgi apparatus in retrograde traffic of the biosythetic pathway, endocytosis and Golgi dis- and reorganization under cellular stress. A variety of publications concerning this subject appeared over the years ${ }^{3-11}$. Nevertheless, our interest focused also on functional three-dimensional ultrastructural aspects of cultured endothelial cells and platelets. In this regard, we will discuss research in this field using ET, preceded by a short explanation of the ET technique.

\section{Explanation of the ET Technique}

ET is carried out using a transmission electron microscope (TEM) with or without a scanning device or a scanning electron microscope equipped with a high voltage gun ( $\geq 200 \mathrm{KV}$ acceleration voltage) in order to penetrate semithin sections (maximal thickness: 1 $\mu \mathrm{m}$ using a STEM device ${ }^{12,13}, 300 \mathrm{~nm}$ without scanning device). Original principles of this method have been summarized by Koster's research group ${ }^{2}$. The TEM must be furnished with a eucentric goniometer that not only allows tilting in the $\mathrm{x} / \mathrm{y}$ axis but also an adjustment of the $\mathrm{z}$ axis reducing the vertical displacement and the amount of focusing needed at each tilt position (autofocus), thus limiting changes in magnification (Fig. 1). ET requires tilting of a semithin section mounted on a copper grid, inserted into a high tilt holder and the acquisition of a tilt series. The holder allows tilting of maximal $-70^{\circ}$ to $+70^{\circ}$. This implicates a disadvantage of this method, the loss of information over the range of $60^{\circ}$ (missing wedge) which can be compensated partially to a so-called missing cone by rotation of the grid around $90^{\circ}$ followed by a $2^{\text {nd }}$ tilt series. A tilt series is composed of a stack of digital images, acquired by a CCD camera starting from the minus to the plus end (in biological preparations usually $-65^{\circ}$ to $+65^{\circ}$ with an increment of $1^{\circ}$ ). The individual images are coarsely aligned and the region of interest (ROI) is centered. This requires a complex acquisition software, allowing to compensate mechanical aberrations and providing that the ROI of every tilt image is in focus and that the eucentric height is ensured (the ROI is placed at the crosspoint of the electron beam and the tilting axis) as explained in Fig. 1.

In a $2^{\text {nd }}$ step, the image of the tilt series is again subjected to a course alignment by cross-correlation with subsequent

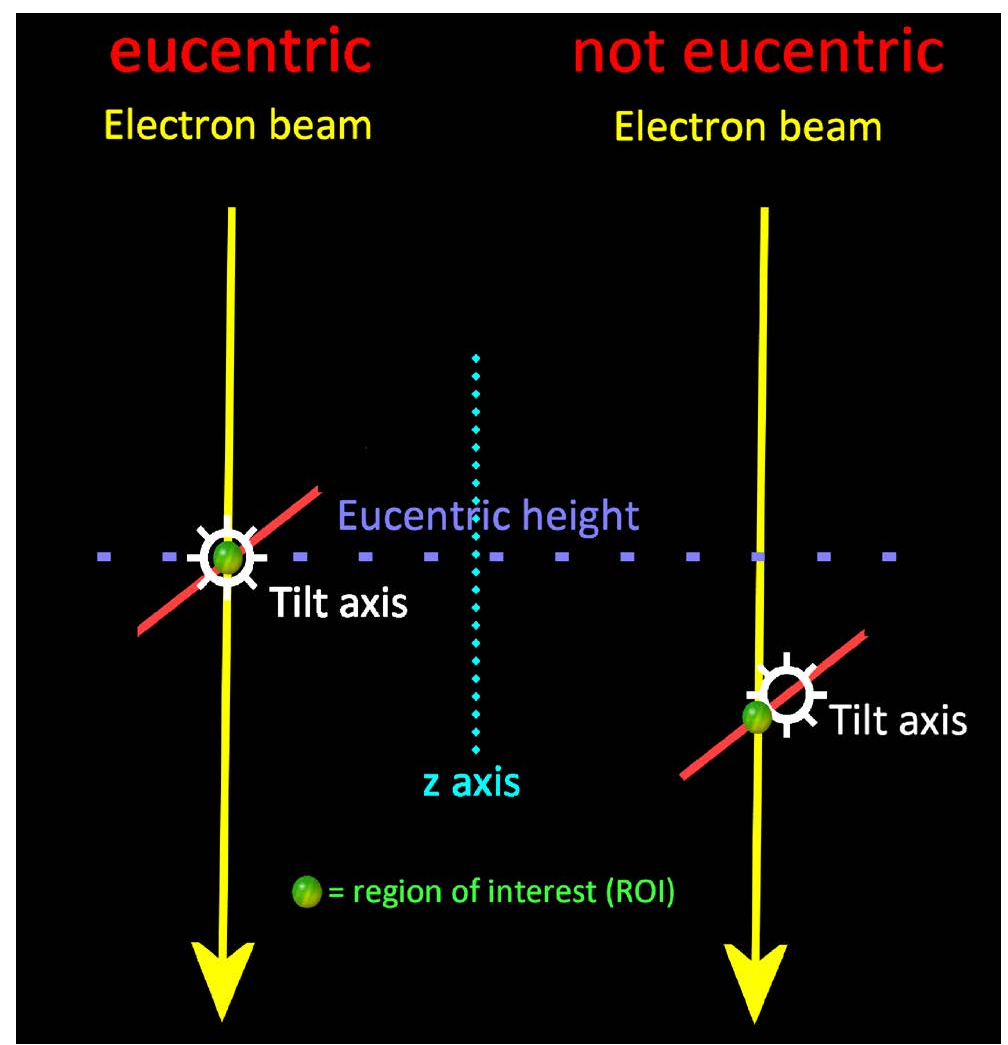

Fig. 1: Eucentric height: The ROI is placed at the crosspoint of the electron beam and the tilting axis. This procedure prevents that the ROI disappears during tilting and enables to perform autofocussing. 
finealignment, guided by the help of fiducial markers $(10 \mathrm{~nm}$ gold particles applied to the surface of the semithin section before starting ET). The final step is the reconstruction process and the creation of a virtual volume, composed of very thin (1-2 $\mathrm{nm}$ ) virtual slices. Usually, alternative reconstruction methods based on different algorithms can be applied: the weighted back projection (WPB) ${ }^{14}$, the simultaneous itinerative reconstruction technique (SIRT) ${ }^{15}$ and the algebraic reconstruction technique (ART) ${ }^{16}$. Loss of structural information using WPB due to missing wedge or missing cone artifacts as well as problems caused by shadowing effects can be partially compensated using SIRT or ART with the disadvantage of long lasting calculation times. The discrete algebraic reconstruction technique (DART) uses prior knowledge concerning the discrete number of grey levels of the reconstructed object ${ }^{17}$. In this publication, the use of SIRT and DART are compared. Some of the respective ET reconstruction software packages can be downloaded free of charge such as the IMOD package ${ }^{18}$ or the TOM toolbox ${ }^{19}$.

Chemical fixation using aldehydes and dehydration alters structural details, above all, at higher magnification. Therefore, cryo methods, such as high pressure fixation followed by cryosubstitution ${ }^{10,20}$ or frozen-hydrated specimens, transferred to the TEM via a cryostage allow a more realistic ultrastructural view ${ }^{21}$. Nevertheless, the operator assigns a subjective interpretation to each profile supported by a correlative view of several aspects of ultrathin sections of the respective area.

New advanced techniques using scanning electron microscopes have been developed and are permanently refined, such as the focused ion beam tomography (FIB-SEM tomography) ${ }^{22,23}$, a combination of FIB-SEM tomography with $\mathrm{ET}^{24,25}$, serial block face scanning electron microscopy (SBEM) ${ }^{26,27}$ and the array tomography ${ }^{28,29}$. All these methods complement each other; their advantages and limitations are summarized in $^{30}$.

\section{Endothelial Cells}

The endothelium represents the border between the blood or lymph and the surrounding tissue. The microcirculation at the tissue level is regulated by small arterioles sphincters as well as capillary by pericytes on capillaries. In addition, it regulates systemic blood pressure by signals directed toward smooth muscle cells of the arterial vascular wall. The interaction with cells and extracellular vesicles of the innate as well as with the adaptive immune systems plays a significant role in health and disease ${ }^{31}$. An important function concerns the maintenance of hemostasis. Healthy endothelial cells express anti-platelet and anti-coagulant agents preventing platelet aggregation and fibrin formation, respectively. After activation of the endothelium due to vessel injury, a chain reaction of pro- inflammatory and wound-healing responses occur by the formation of a primary platelet plug followed by activation of the coagulation system to form a stable fibrin clot $^{32}$. A significant role in this process plays the formation of endothelium-specific Weibel-Palade bodies (WPB). They represent cigar-like organelles with an average size of $0.1 \mathrm{x}$ $2 \mu \mathrm{m}$ containing parallel tubules with a diameter of about $20 \mathrm{~nm}$, composed of multimerized von Willebrand factor (vWF). Beside vWF and vWF propeptide, WPB contain a variety of adhesion molecules and cyto- and chemokines such as factor VIII, CD62P, IL-8, eotaxin-3, endothelin, CD63/ lamp3, angiopoietin-2, $\alpha 1,3$-fucosyltransferase VI, tissue plasminogen activator, and osteoprotegerin. The special organization of WPB has been elucidated by Valentijn et al. ${ }^{33}$ in human umbilical vein endothelial cells (HUVEC) using ET. Upon activation - for instance, due to injury WPB are exocytosed and extracellularly cleaved into long filaments by the metalloprotease ADAMTS-13. The authors demonstrated a regular twisting of the microtubules and in cross sections an electron-dense dot in their center. They suggest that the regular twisting of the tubules may reflect a higher order of compaction or a "spring loading mechanism" in respect to WPB exocytosis. They describe an evenly spaced homogeneous matrix around the tubules, probably representing an underlying scaffold of structural proteins. In addition, irregular shapes of WPB could be demonstrated that agreed with our own findings in investigations on end othelial progenitor cells (EPC) derived from human umbilical cord stem cells ${ }^{1,34,35}$. As shown in Fig. 2 , the transition of EPC in mature endothelial cells could be demonstrated by the occurrence of complete WPB in the cells (Fig. 2a). The presence of vWF in the TGN has been shown using fluorolabeled monoclonal antibodies against TGN-46 (orange) and vWF (green) in a 3D reconstruction of slices using a laser confocal microscope (LSM; Fig. 2c). Fig. $2 b$ shows the budding of TGN with newly formed WPB and Fig. 2d an ET model from a mature and a newly formed WPB. Since a conventional fixation and dehydration protocol has been used in this model, minimal distortions cannot be excluded. A more recent publication on WPB in HUVEC focused also on this issue ${ }^{36}$. The authors applied correlative microscopical techniques using LSM, ET and volume scanning electron microscopy. They showed the existence of multiple connections with the Golgi apparatus during biogenesis of WPB, where the formation of tubular structures occurs in the WPB lumen, provided by a clathrin scaffold. As postulated in another publication ${ }^{37}$ intracellular and extracellular assembly of FVIII-vWF complexes seem to involve different mechanisms, influencing the binding of PLT on activated endothelial cells. An interesting article ${ }^{38}$ elucidated the communication of endothelium and smooth muscle cells via endothelial projections from mesenteric arteries of golden Syrian hamsters. ET visualized projections crossing the internal elastic lamina. The 

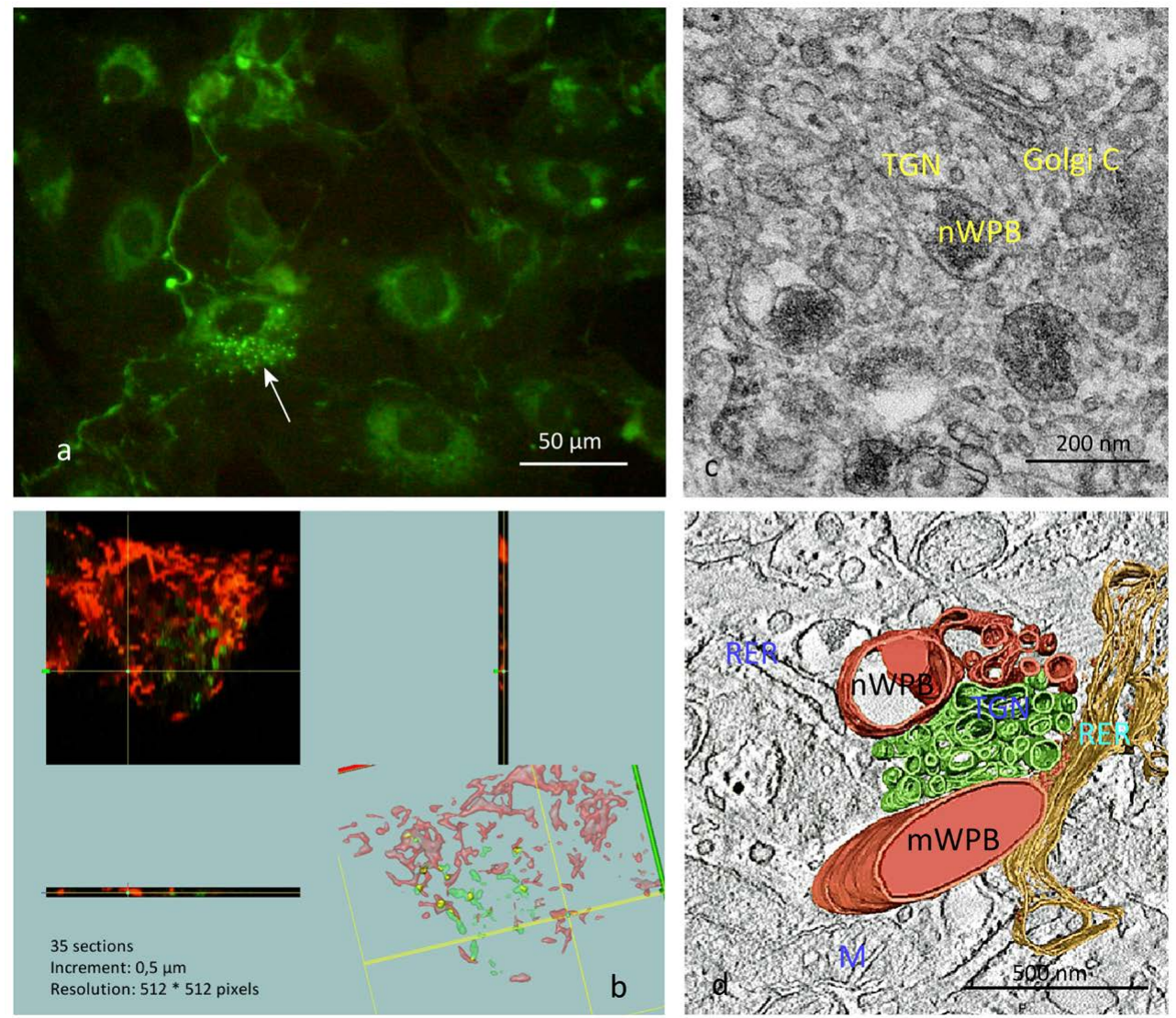

Fig. 2a: Transition of EPC into mature endothelial cells demonstrated by the occurrence of complete WPB in the cells; fluorescence microscopy using a FITC-conjugated monoclonal antibodies against VWF. Fig. 2b: Presence of vWF in the TGN is shown in a 3D imaging in a LSM using a TRITC-conjugated monoclonal antibodies against TGN-46 (red) and a FITC-conjugated monoclonal antibodies against vWF (green) (LSM was performed using a Leica TCS 4D confocal microscope, Leica Microsystems Heidelberg GmbH, Heidelberg, Germany. 3D reconstruction of LSM slices was performed by using the software package resolve RT, Amira 5.3 software (Mercury Computer Systems, Merignac, Cedex, France). Fig. 2c: shows the budding of TGN with newly formed WPB and Fig. 2d: an ET model from a mature (mWPB) and a newly formed WPB (nWPB). RER = rough endoplasmic reticulum, $M=$ mitochondrium, TGN = trans golgi network. The TGN is colored in green or red, where it is thought to be connected with the WPB (also in red). The cross-sectioned microtubular structures inside of the WPB are not shown. ET was carried out from $200-300 \mathrm{~nm}$ semithin sections conventionally prepared (aldehyde-fixed, ethanol-dehydrated and embedded in Epon). Series of tilted images within an angular range of $-65^{\circ}$ to $+65^{\circ}$ and a tilt increment of $1^{\circ}$ were recorded automatically by the Xplore 3D software (FEI company) using a 1k Gatan slow-scan CCD camera (chip size: $1.024 \times 1.024$ pixels. Reconstructions were performed using the IMOD software (Boulder Laboratory for 3D Electron Microscopy of Cells, University of Colorado, USA).

authors suggest that these cell contacts provide dynamic sites for signal regulation of contraction of smooth muscle cells. By using fluorescence microscopy and ET, the uptake and the intracellular transport in HUVEC and EPC involving the endoplasmic reticulum, the Golgi apparatus and the endo- and lysosomal system ${ }^{7,39}$ has been monitored. In these investigations, correlative microscopy, including the application of EM visualization of fluorescent signals in cellular compartments and organelles by means of DABphotoconversion ${ }^{4,6}$ has been applied.

\section{Platelets (PLT)}

PLT, originating from megakaryocytes, share similarities in respect to their constituents ${ }^{40}$. Representing only cell fragments, they surprisingly exhibit a complex ultrastructural morphology including a peripheral microtubular coil (MTC), different kinds of granules, the open canalicular system (OCS), the dense tubular system (DTS) mitochondria (MIT), glycogen (Gly) and ribosomes. ET and cryo-ET allowed an impressive 3D view of these organelles, leading to a better understanding of PLT function. The publication of van Nispen tot Pannerden and coworkers ${ }^{41}$ represents an outstanding contribution to this issue. This research group could show that OCS and DTS are highly intertwined, forming close associations with specialized membrane domains. Concerning the $\alpha$-granules, they demonstrated several species with different shapes, such as spherical, elongated, vesiculated and tabulated forms. One subpopulation of granules exhibited crystalline cross striations. The 3D reconstruction of whole vitrified PLT revealed the spatial extensions of these organelles. 

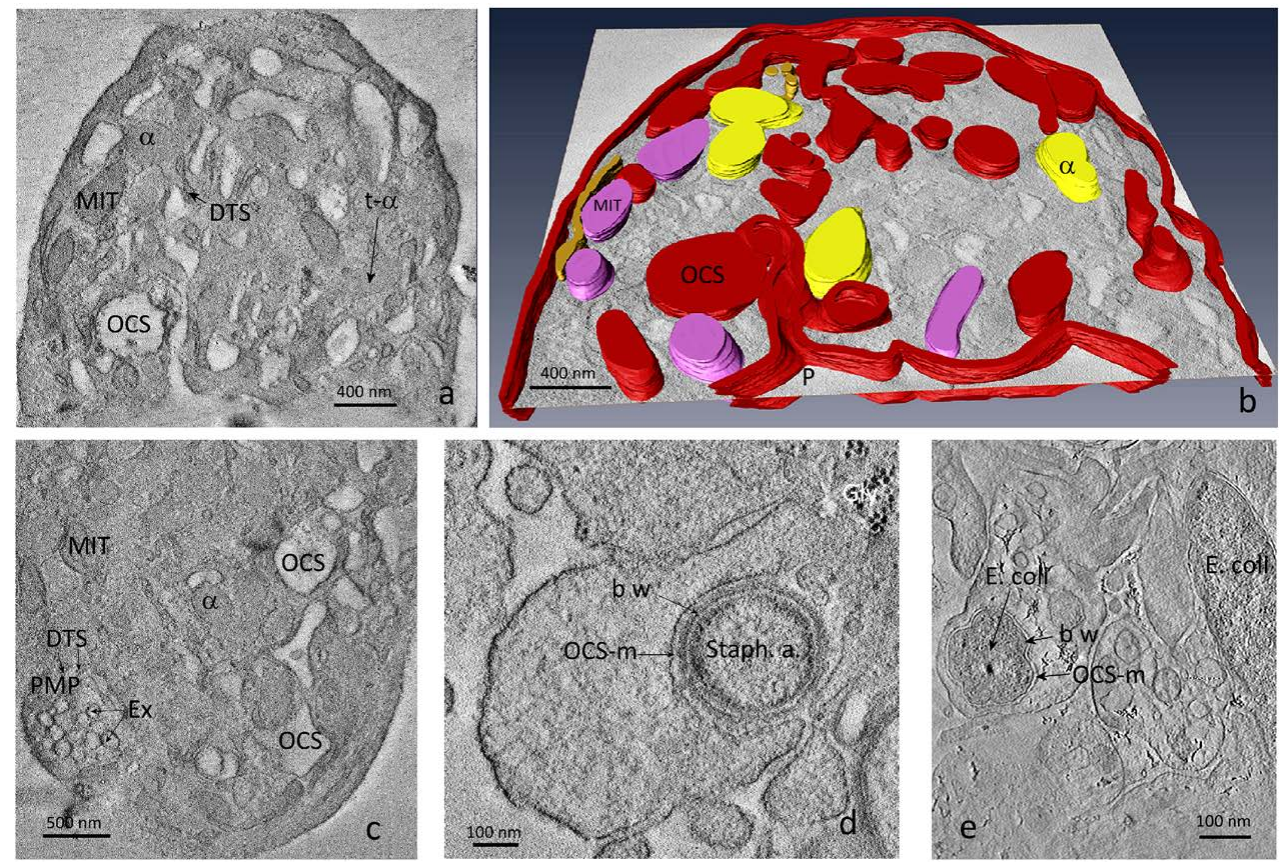

Fig. 3: ET from PLT. Figs. 3a,c: Virtual slices showing the open canalicular system OCS with pores (P) (connection with the extracellular milieu), MIT, $\alpha$-granules $(\alpha)$, tubular granules with few filaments (t- $\alpha$ ), glycogen deposits (Gly) and the dense tubular system DTS. Fig. 3b: Model from ET reconstruction shown in Fig. 3a. Fig. 3c: Virtual slice showing the delivery of exosomes (Ex) in a multivesicular body. In Fig. 3d: Uptake of a Staphylococcus aureus (Staph. a.) into the OCS, Fig. 3e: Complete engulfment of an Escherichia coli bacterium (E. coli). OCS-m = OCS membrane, $\mathrm{b}$ w = bacterial wall. ET was carried out as described in Fig. 2.

The OCS is thought to originate from the demarcation membrane system (DMS) of megakaryocytes. In an interesting publication, using correlative microscopy, involving LSM, ET and FIB-SEM, the following steps of DMS biogenesis are postulated ${ }^{42}$ : (1) focal membrane assembly at the cell periphery; (2) plasma membrane invagination and formation of a perinuclear pre-DMS; (3) expansion through membrane delivery from Golgi complexes; and (4) ERmediated lipid transfer. The research group of Pokrovskaya ${ }^{43}$ demonstrated by using STEM tomography that in resting PLT, $\alpha$-granules and the OCS remain separate units. Tubular granules, representing about $4 \%$ in resting PLT, increase for more than 10-fold during ongoing PLT activation. Our own investigations elucidated the interaction of bacteria with PLT in platelet concentrates (PLTC) and the formation of platelet microparticles ${ }^{44}$. Since there is a minimal risk of bacterial contamination in non-pathogen-inactivated PLTC, we investigated the interaction of bacteria and PLT. Using the tracer ruthenium red, we could show that PLT from PLTC, spiked with Staphylococcus aureus or Escherichia coli, are able to phagocytose and kill them. In addition, an enhanced formation of platelet microparticles (PMP) was demonstrated. Two species of PMP can be found: larger particles (300-500 $\mathrm{nm}$ in diameter) and exosomes, derived from multivesicular bodies (50-80 $\mathrm{nm}$ in diameter). These results are illustrated in Fig. 3. Extracellular vesicles in fresh plasma and PMP in PLT-rich plasma were also investigated by cryo-EM and cryo-ET ${ }^{45}$. In this context, an interesting article of a research group ${ }^{46}$ described the macromolecular architecture of PMP, visualized using cryo-ET.

In conclusion, there is an enhanced scientific interest in bringing together results from biochemistry and structural biology. In this respect, the correlative microscopy bridges between light microscopy, enabling to investigate vital processes in cells and tissues, and EM, visualizing only snapshots of fixed or frozen specimens but providing high resolution. New techniques including cryomethods allow locating even macromolecular structures inside of cells.

\section{References}

1. Neumüller J. Electron tomography-a tool for ultrastructural $3 \mathrm{D}$ visualization in cell biology and histology. Wiener medizinische Wochenschrift. 2018; 168: 322-9.

2. Koster AJ, Grimm R, Typke D, et al. Perspectives of molecular and cellular electron tomography. Journal of structural biology. 1997; 120: 276-308.

3. Pavelka M, Neumüller J, Ellinger A. Retrograde traffic in the biosynthetic-secretory route. Histochemistry and cell biology. 2008; 129: 277-88.

4. Meißlitzer-Ruppitsch C, Vetterlein M, Stangl H, et al. Electron microscopic visualization of fluorescent signals in cellular compartments and organelles by means of DAB-photoconversion. Histochemistry and cell biology. 2008; 130: 407-19.

5. Meißlitzer-Ruppitsch C, Röhrl C, Ranftler C, et al. The ceramideenriched trans-Golgi compartments reorganize together with 
other parts of the Golgi apparatus in response to ATP-depletion Histochemistry and cell biology. 2011; 135: 159-71.

6. Meißlitzer-Ruppitsch C, Röhrl C, Ranftler C, et al. Photooxidation technology for correlative light and electron microscopy. Methods in molecular biology. 2013; 931: 423-36.

7. Röhrl C, Pagler TA, Strobl W, et al. Characterization of endocytic compartments after holo-high density lipoprotein particle uptake in HepG2 cells. Histochemistry and cell biology. 2010; 133: 261-72.

8. Ranftler C, Meißlitzer-Ruppitsch C, Neumüller J, et al. Golgi apparatus dis- and reorganizations studied with the aid of 2-deoxy-D-glucose and visualized by 3D-electron tomography. Histochemistry and cell biology. 2017; 147: 415-38.

9. Marsh BJ, Pavelka M. Viewing Golgi structure and function from a different perspective--insights from electron tomography. Methods in cell biology. 2013; 118: 259-79.

10. Ellinger A, Vetterlein M, Weiss $C$, et al. High-pressure freezing combined with in vivo-DAB-cytochemistry: a novel approach for studies of endocytic compartments. Journal of structural biology. 2010; 169: 286-93.

11. Martinez-Martinez N, Martinez-Alonso E, Tomas M, et al. A new insight into the three-dimensional architecture of the Golgi complex: Characterization of unusual structures in epididymal principal cells. PloS one. 2017; 12: e0185557.

12. Aoyama K, Nagano K, Mitsuoka K. Optimization of STEM imaging conditions for cryo-tomography. Microscopy. 2017; 66: 212-6.

13. Walther P, Bauer A, Wenske N, et al. STEM tomography of highpressure frozen and freeze-substituted cells: a comparison of image stacks obtained at $200 \mathrm{kV}$ or $300 \mathrm{kV}$. Histochemistry and cell biology. 2018; 150: 545-56.

14. Fernandez JJ, Lawrence AF, Roca J, et al. High-performance electron tomography of complex biological specimens. Journal of structural biology. 2002; 138: 6-20.

15. Zhang F, Zhang J, Lawrence A, et al. BSIRT: a block-iterative SIRT parallel algorithm using curvilinear projection model. IEEE transactions on nanobioscience. 2015; 14: 229-36.

16. Marabini R, Herman GT, Carazo JM. 3D reconstruction in electron microscopy using ART with smooth spherically symmetric volume elements (blobs). Ultramicroscopy. 1998; 72: 53-65.

17. Goris B, Roelandts T, Batenburg KJ, et al. Advanced reconstruction algorithms for electron tomography: from comparison to combination. Ultramicroscopy. 2013; 127:40-47.

18. Kremer JR, Mastronarde DN, McIntosh JR. Computer visualization of three-dimensional image data using IMOD. Journal of structural biology. 1996; 116: 71-6.

19. Nickell S, Förster F, Linaroudis A, et al. TOM software toolbox acquisition and analysis for electron tomography. Journal of structural biology. 2005; 149: 227-34.

20. McDonald KL, Auer M. High-pressure freezing, cellular tomography, and structural cell biology. BioTechniques. 2006; 41: 137, 9, 41 passim.

21. Irobalieva RN, Martins B, Medalia O. Cellular structural biology as revealed by cryo-electron tomography. Journal of cell science. 2016; 129: 469-76.

22. Kizilyaprak C, Bittermann AG, Daraspe J, et al. FIB-SEM tomography in biology. Methods in molecular biology. 2014; 1117: 541-58.

23. Villa E, Schaffer M, Plitzko JM, et al. Opening windows into the cell: focused-ion-beam milling for cryo-electron tomography. Current opinion in structural biology. 2013; 23: 771-7.

24. Rigort, Plitzko JM. Cryo-focused-ion-beam applications in structural biology. Archives of biochemistry and biophysics. 2015; 581: 122-30.
25. Wagner J, Schaffer M, Fernandez-Busnadiego R. Cryo-electron tomography-the cell biology that came in from the cold. FEBS letters. 2017; 591: 2520-33.

26. Zankel A, Wagner J, Pölt P. Serial sectioning methods for 3D investigations in materials science. Micron. 2014; 62: 66-78.

27. Denk W, Horstmann H. Serial block-face scanning electron microscopy to reconstruct three-dimensional tissue nanostructure. PLoS biology. 2004; 2: e329.

28. Micheva KD, Smith SJ. Array tomography: a new tool for imaging the molecular architecture and ultrastructure of neural circuits. Neuron. 2007; 55: 25-36.

29. Oberti D, Kirschmann MA, Hahnloser RH. Projection neuron circuits resolved using correlative array tomography. Frontiers in neuroscience. 2011; 5: 50 .

30. Kizilyaprak C, Daraspe J, Humbel BM. Focused ion beam scanning electron microscopy in biology. Journal of microscopy. 2014; 254: 109-14.

31. Khaddaj Mallat R, Mathew John C, Kendrick DJ, et al. The vascular endothelium: A regulator of arterial tone and interface for the immune system. Critical reviews in clinical laboratory sciences. 2017; 54: 458-70.

32. Yau JW, Teoh H, Verma S. Endothelial cell control of thrombosis. BMC cardiovascular disorders. 2015; 15: 130

33. Valentijn KM, Valentijn JA, Jansen KA, et al. A new look at WeibelPalade body structure in endothelial cells using electron tomography. Journal of structural biology. 2008; 161: 447-58.

34. Neumüller J, Neumüller-Guber SE, Lipovac M, et al. Immunological and ultrastructural characterization of endothelial cell cultures differentiated from human cord blood derived endothelial progenitor cells. Histochemistry and cell biology. 2006; 126: 649-64.

35. Neumüller J, Ellinger A. Secretion and endocytosis in endothelial cells. In: Mironov A.A and Pavelka M, (ed.). The Golgi Apparatus. Springer Vienna. 2008; 520-34.

36. Mourik MJ, Faas FG, Zimmermann H, et al. Content delivery to newly forming Weibel-Palade bodies is facilitated by multiple connections with the Golgi apparatus. Blood. 2015; 125: 3509-16.

37. Bouwens EA, Mourik MJ, van den Biggelaar M, et al. Factor VIII alters tubular organization and functional properties of von Willebrand factor stored in Weibel-Palade bodies. Blood. 2011; 118: 5947-56.

38. Maarouf N, Sancho M, Fürstenhaupt T, et al. Structural analysis of endothelial projections from mesenteric arteries. Microcirculation. $2017 ; 24$.

39. Srisen K, Röhrl C, Meißlitzer-Ruppitsch C, et al. Human endothelial progenitor cells internalize high-density lipoprotein. PloS one. 2013; 8: e83189.

40. Han ZC, Caen JP. Are megakaryocytes and endothelial cells sisters? The Journal of laboratory and clinical medicine. 1993; 121: 821-5.

41. van Nispen tot Pannerden H, de Haas F, Geerts W, et al. The platelet interior revisited: electron tomography reveals tubular alpha-granule subtypes. Blood. 2010; 116: 1147-56.

42. Eckly A, Heijnen H, Pertuy F, et al. Biogenesis of the demarcation membrane system (DMS) in megakaryocytes. Blood. 2014; 123: 92130.

43. Pokrovskaya ID, Aronova MA, Kamykowski JA, et al. STEM tomography reveals that the canalicular system and alpha-granules remain separate compartments during early secretion stages in blood platelets. Journal of thrombosis and haemostasis : JTH. 2016; 14: 572 84 .

44. Neumüller J, Ellinger A, Wagner T. Transmission Electron 
Neumüller J, Wagner T. Electron Tomography Contributing to Ultrastructural Research in Vas-

Microscopy of Platelets from Apheresis and Buffy-Coat-Derived

Platelet Concentrates. In: Maaz K, (ed.). The Transmission Electron

Microscope - Theory and Applications. London: Intech-Open Science/

Open Minds. 2015; 255-84.

45. Yuana Y, Koning RI, Kuil ME, et al. Cryo-electron microscopy of extracellular vesicles in fresh plasma. Journal of extracellular vesicles. $2013 ; 2$.

46. Tamir A, Sorrentino S, Motahedeh S, et al. The macromolecular architecture of platelet-derived microparticles. Journal of structural biology. 2016; 193: 181-7. 